\title{
Balkan Dry Grasslands Database
}

\author{
Kiril Vassilev, Zora Dajić Stevanović, Renata Cušterevska, Erwin Bergmeier \& Iva \\ Apostolova
}

\begin{abstract}
Dry grasslands on the Balkan Peninsula are characterized by a rich diversity. They include syntaxa mostly referred to Festuco-Brometea. Relevés from Bulgaria, Serbia, Macedonia, Kosovo and Greece are included in this database aiming to cover geographically the central part of the peninsula. Significant parts of the data have been collected in the recent years. More than 150 literature sources have been collected and their data were digitized. The database is in TURBOVEG format. It includes 4,520 relevés. All of them contain ecological data as well as quantitative estimates for the species. About $15 \%$ of the samples contain cryptogams. Some of the relevés overlap with national or private databases. The database is built on collaboration among scientists dealing with vegetation classification from all countries in the Central Balkans. This report describes the available content in the Balkan Dry Grasslands Database (GIVD ID EU-00-013).
\end{abstract}

Keywords: Festuco-Brometea; relevé; steppe; vegetation.

\begin{tabular}{|c|c|}
\hline GIVD Database ID: EU-00-013 & Last update: $2012-05-06$ \\
\hline \multicolumn{2}{|l|}{ Balkan Dry Grasslands Database } \\
\hline \multicolumn{2}{|c|}{$\begin{array}{l}\text { Scope: Relevés from dry grasslands (Festuco-Brometea, Helianthemetea guttati, Koelerio-Corynephoretea incl. Sedo-Scleranthetea and } \\
\text { Festucetea vaginatae) collected from the Balkan peninsula. The plot size varies between } 16-100 \mathrm{~m}^{2} \text {. }\end{array}$} \\
\hline Status: emerging & Period: $1947-2011$ \\
\hline \multicolumn{2}{|c|}{ Database manager(s): Kiril Vassilev (kiril5914@abv.bg) } \\
\hline \multicolumn{2}{|c|}{ Owner: Kiril Vassilev, Zora Dajić Stevanović, Renata Cušterevska, Erwin Bergmeier, Iva Apostolova (private) } \\
\hline \multicolumn{2}{|l|}{ Web address: $[N A]$} \\
\hline Availability: according to a specific agreement & Online upload: no \\
\hline Database format(s): TURBOVEG & Export format(s): TURBOVEG \\
\hline \multicolumn{2}{|l|}{ Publication: [NA] } \\
\hline Plot type(s): normal plots; nested plots & Plot-size range: $16-100 \mathrm{~m}^{2}$ \\
\hline Non-overlapping plots: 4,520 & Estimate of existing plots: $[\mathrm{NA}]$ \\
\hline Total plot observations: 4,520 & Number of sources: 150 \\
\hline \multicolumn{2}{|c|}{ Countries: BG: $45.0 \%$; GR: 5.0\%; MK: 13.0\%; RS: $37.0 \%$} \\
\hline \multicolumn{2}{|c|}{ Forest: $[\mathrm{NA}]$ - Non-forest: $[\mathrm{NA}]$} \\
\hline \multicolumn{2}{|c|}{ Guilds: all vascular plants: $100 \%$; bryophytes (terricolous or aquatic): $15 \%$; lichens (terricolous or aquatic): $10 \%$} \\
\hline \multicolumn{2}{|c|}{ Environmental data: altitude: $96 \%$; slope aspect: $85 \%$; slope inclination: $89 \%$; soil pH: $18 \%$} \\
\hline \multicolumn{2}{|l|}{ Performance measure(s): [NA] } \\
\hline \multicolumn{2}{|c|}{ Geographic localisation: GPS coordinates (precision $25 \mathrm{~m}$ or less): $31 \%$} \\
\hline $\begin{array}{l}\text { Sampling periods: } 1940-1949: 1.0 \% ; 1950-195 \\
30.0 \% ; 2010-2019: 32.0 \%\end{array}$ & 59: 7.0\%; 1960-1969: 7.0\%; 1970-1979: 14.0\%; 1980-1989: 5.0\%; 1990-1999: 4.0\%; 2000-2009: \\
\hline Information as of 2012-07-12; fur & Irther details and future updates available from http://www.givd.info/ID/EU-00-013 \\
\hline
\end{tabular}

Kiril Vassilev* (kiril5914@abv.bg), Iva Apostolova (iva.apostolova@gmail.com)

Plant and Fungi Diversity and Resources, Institute of Biodiversity and Ecosystem Research, 23 Acad. Georgi Bonchev, 1113 Sofia, BULGARIA

Zora Dajić Stevanović (dajic@agrif.bg.ac.rs)

Department of Botany, Belgrade University, SERBIA

Renata Cušterevska (renatapmf@yahoo.com)

University of Skopje, MACEDONIA

Erwin Bergmeier (erwin.bergmeier@bio.uni.goettingen.de)

University of Göttingen, GERMANY

*Corresponding author 